\title{
Estrategias de educación médica continua
}

\author{
Amanda Galli, Carlos Soler, Daniel Flichtentrei, Ricardo Mastandueno
}

Resumen. Para ejercer apropiadamente a lo largo de su vida profesional, los médicos han de involucrarse en alguna forma de educación continua. La educación médica continua no se limita a la educación formal desarrollada mediante tal o cual método educativo, sino que comprende experiencias de muy diversa índole, formales e informales, tales como los encuentros con colegas, el autoaprendizaje y la educación a distancia. El propósito de esta publicación es presentar los resultados obtenidos al explorar las estrategias y recursos mas frecuentemente utilizados por los profesionales médicos para mantenerse actualizados. Se implementó una encuesta electrónica de cinco preguntas cerradas con varias opciones de respuesta. Las preguntas indagaban sobre: lugar de trabajo, frecuencia con la que experimenta 'necesidad' de actualizar sus conocimientos, modalidades y recursos utilizados. Se obtuvieron 9.851 respuestas. Las modalidades más frecuentemente mencionadas fueron asistencia a cursos presenciales, a congresos y seminarios, y participación en actividades hospitalarias. Las revistas publicadas por sociedades científicas y las guías clínicas son las fuentes de información señaladas con mayor frecuencia. Los recursos disponibles en Internet fueron mencionados por menos del $20 \%$ de los profesionales que respondieron. Se encontraron algunas diferencias entre las modalidades y recursos que utilizan los médicos que trabajan sólo en su consulta y los que atienden en un hospital.

Palabras clave. Educación médica continua. Recursos para la actualización. Utilización de Internet.

\section{Continuing medical education strategies}

Summary. To properly exercise throughout his professional life, doctors have to engage in some form of continuing education. Continuing medical education is not limited to formal education, includes very diverse experiences, formal and informal such as meetings with colleagues, self-learning and distance education. The purpose of this paper is to present the results to explore strategies and resources used by medical professionals to keep up to date. Implemented an electronic survey of 5 questions with several response options; explored questions about: workplace, frequency experiences 'need' to update their medical knowledge, methods and resources used more frequently. 9.851 answers were obtained. The most frequently mentioned modalities were assistance to courses, conferences and seminars and participating in activities in the hospital. Journals published by scientific societies and clinical guidelines are information sources referred to most frequently. The resources available on the internet were mentioned by less than $20 \%$ of the professionals who responded. There are some differences between the methods and resources used by doctors who work only in their offices and those who attend at hospital.

Key words. Continuing medical education. Resources for updating. Using internet.

\section{Introducción}

La educación médica continua puede definirse como el conjunto de acciones y recursos dirigidos a cambiar la conducta de los profesionales para mejorar los resultados de su actuación con los pacientes; también se define como toda actividad que realiza el médico para seguir aprendiendo una vez finalizada su formación universitaria [1].

El desarrollo científico y tecnológico, las demandas de la nueva ética y el desarrollo socioeconómico afectan de forma continua a la profesión médica; el profesional debe conservar un espíritu permanentemente inquisitivo y desarrollar una actividad de actualización continua para sostener un nivel de desempeño aceptable.

La educación continua dista de ser una novedad. Sócrates y Platón consideraban la educación como un proceso de toda la vida. Osler [2], en 1900, publicó una nota sobre la importancia de la educación continua y, en 1961, el tema de la Segunda Conferencia Mundial de Educación Médica fue 'Medicina, un estudio de toda la vida'. Todavía hoy, la educación continua constituye el único tratamiento co-
Sociedad Argentina de Investigación y Desarrollo en Educación Médica, SAIDEM (A. Galli, C. Soler). IntraMed (D. Flichtentrei, R. Mastandueno). Buenos Aires, Argentina.

Correspondencia: Lic. Amanda Galli. SAIDEM. Juncal,

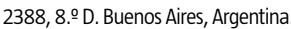

E-mail:

amandaelisagalli@gmail.com

Nota:

Las tablas con los datos correspondientes al análisis comparativo se hallan disponibles en www.saidem.org.ar.

Conflicto de intereses: No declarado.

Competing interests: None declared.

(C) 2015 FEM 
nocido para prevenir la obsolescencia progresiva de la competencia profesional.

La educación médica continua es amplia, no se limita a la educación formal desarrollada mediante tal o cual método educativo; comprende una gama de experiencias de muy diversa índole, incluye los encuentros entre colegas, el autoaprendizaje y la educación a distancia. Alfonso Mejía [3] afirmó que 'la educación inicial, independientemente de su duración, no garantiza un ejercicio profesional idóneo indefinidamente. Capacita sólo para comenzar una carrera o empleo y aporta los cimientos para continuar la educación durante toda la vida profesional de un individuo. Sin educación continua, la competencia decrece progresiva e inexorablemente'.

Para ejercer apropiadamente a lo largo de su vida profesional, los médicos han de estar al día, lo cual implica involucrarse en alguna forma de educación continua. La Federación Mundial de Educación Médica (WFME) [4] sostiene que 'el antiguo término «educación médica continuada» ha sido substituido por el de «desarrollo profesional continuo». El nuevo término refleja el contexto más amplio en que se encuentra esta fase de la educación médica e implica que la responsabilidad de practicar el desarrollo profesional continuo recae en la profesión y en el médico como individuo'.

El propósito de esta comunicación es presentar los resultados obtenidos al explorar las estrategias que utilizan los profesionales médicos para mantenerse actualizados.

\section{Materiales y métodos}

Se implementó una encuesta electrónica de cinco preguntas cerradas con varias opciones de respuesta. Las preguntas indagaban sobre:

- Lugar de trabajo: en hospital/sanatorio con ingreso o sólo en su consulta (atención ambulatoria) (Tabla I).

- Frecuencia con la que experimenta 'necesidad' de actualizar sus conocimientos médicos (Tabla II).

- Modalidades que utiliza más frecuentemente para actualizar sus conocimientos (Tablas III y IV).

- Fuentes de información que utiliza más frecuentemente (Tablas V y VI).

- Recursos de Internet que utiliza más frecuentemente (Tabla VII).

La encuesta estuvo disponible en http://www.intramed.net/ durante 45 días. Las respuestas fueron anónimas y voluntarias. Se utilizó el test de chi al cuadrado para analizar si había diferencia en las estra- tegias y recursos que utilizaban los profesionales según el lugar de trabajo.

\section{Resultados}

Durante marzo-abril de 2013 se recibieron 9.851 respuestas: $48 \%$ de Argentina, 17\% de México y el resto de otros países de América del Sur; $56 \%$ de sexo masculino.

El 50\% se ubicaba en el rango de 40-50 años de edad, y el 35\%, en el rango de 20-30 años.

El 99,69\% eran médicos. Predominaba la especialidad clínica médica (49\%), seguida de pediatría (12\%).

Respecto a las modalidades que utilizaban más frecuentemente para actualizar sus conocimientos (Tabla III), los profesionales que marcaron dos opciones generaron 28 combinaciones diferentes; las más frecuentes resultaron las que se indican en la tabla IV.

Respecto a las principales fuentes de información (Tabla V), los profesionales que marcaron dos opciones generaron distintas combinaciones; las más frecuentes se muestran en la tabla VI.

\section{Resultados comparativos}

Para analizar si existen diferencias entre las respuestas de los profesionales que atienden sólo en su consulta y los que atienden en el hospital se formaron dos grupos tras eliminar a 1.478 sujetos que informaron trabajar en ambos lugares. El grupo A quedó integrado por 5.010 profesionales que atendían en el hospital, y el grupo B, por 3.363 profesionales que atendían sólo en su consulta, conformando un total de 8.373 sujetos. Al comparar ambos grupos se encontraron las siguientes diferencias y similitudes:

- Los médicos de consulta (grupo B) mayoritariamente tienden a actualizar sus conocimientos frente a los problemas concretos que presenten sus pacientes. Los de hospital también lo hacen, pero en mucha menor proporción $(p=0,0001)$.

- Ambos grupos prefieren las actividades presenciales para la actualización de sus conocimientos.

- Los médicos de consulta (grupo B) asisten a congresos y seminarios más que sus colegas del hospital (grupo A) $(p=0,0001)$.

- En ambos grupos, menos del $10 \%$ ha realizado algún curso online.

- En ambos grupos, el intercambio entre colegas como forma de actualizar conocimientos es señalado por menos del 10\%. Los profesionales de ambos grupos recurren por igual a la consulta con un colega. 
Tabla I. Lugar de trabajo $(n=9.851)$.

\begin{tabular}{lc}
\hline Trabaja en un hospital o sanatorio con ingreso & $5.010(50,9 \%)$ \\
\hline Trabaja sólo en su consulta, atención ambulatoria & $3.363(34,1 \%)$ \\
\hline En ambos & $1.478(15 \%)$ \\
\hline
\end{tabular}

Tabla IV. Combinaciones más frecuentes para actualizar los conocimientos $(n=9.851)$.

\begin{tabular}{lc}
\hline Congresos/cursos presenciales & $1.681(17,6 \%)$ \\
\hline Actividad hospitalaria/cursos presenciales & $1.516(15,3 \%)$ \\
\hline Cursos presenciales/publicación en Internet & $1.284(13,3 \%)$ \\
\hline
\end{tabular}

- Los médicos del grupo A se actualizan participando en actividades tales como pase de sala y ateneos, más que sus colegas del grupo $\mathrm{B}(p=0,0001)$ También utilizan más las guías clínicas y otras publicaciones distribuidas por las autoridades sanitarias $(p=0,0001)$.

- La lectura de revistas científicas, en papel o en Internet, es una modalidad de educación continua más usada por los médicos de consulta $(p=0,0001)$. Los médicos de consulta, más que los de hospital, son usuarios de Internet ( $p=0,0001)$.

- Ambos grupos dicen utilizar Internet como fuente de información (consultan Wikipedia); en el grupo A aparece un mayor uso de PubMed ( $p=$ 0,0001).

- Ambos grupos dicen utilizar la información distribuida por la industria farmacéutica. Los médicos de consulta recurren más que sus colegas de hospital a esta fuente de información $(p=0,0001)$.

\section{Discusión}

Según los datos obtenidos, los médicos reconocen que necesitan buscar información de forma permanente y que esta necesidad surge, fundamentalmente, de la práctica asistencial. Slotnick [5] afirmó que los profesionales aprenden buscando respuesta a los problemas específicos que tienen que afrontar y, ante los problemas específicos o puntuales que plantean los pacientes, recurren a distintas fuentes de información, mientras que en relación a temas o problemas en general, asisten a cursos formales, especialmente en las sociedades científicas.
Tabla II. ¿Con qué frecuencia experimenta 'necesidad' de actualizar sus conocimientos médicos? ( $n=9.851$ )

\begin{tabular}{lc}
\hline Nunca, hace años que no siento necesidad de buscar más información & $10(0,1 \%)$ \\
\hline Casi nunca, los conocimientos que tengo son suficientes para mi práctica médica & $60(0,6 \%)$ \\
\hline Sólo si tengo dudas con algún paciente & $1.318(13,4 \%)$ \\
\hline Con mucha frecuencia, casi todas las semanas & $8.463(85,9 \%)$
\end{tabular}

Tabla III. Marque las dos modalidades que utiliza más frecuentemente para actualizar sus conocimientos.

\begin{tabular}{lc}
\hline Asistir a cursos presenciales en sociedad científica, universidad, colegio médico, industria & 3.632 (36,8\%) \\
\hline Asistir a congresos y jornadas & 3.609 (36,6\%) \\
\hline Participar en la actividad hospitalaria (pase de sala, ateneos, supervisión) & 3.227 (32,8\%) \\
\hline Leer publicaciones en Internet & $2.833(28,8 \%)$ \\
\hline \begin{tabular}{l} 
Leer revistas científicas en papel \\
\hline Intercambiar experiencias entre colegas
\end{tabular} & 661 (8,7\%) \\
\hline $\begin{array}{l}\text { Consultar a un colega amigo } \\
\text { Hacer cursos a distancia con entrega de material impreso en domicilio }\end{array}$ & 0 \\
\hline $\begin{array}{l}\text { El porcentaje, en cada una de las modalidades, se refiere al total de sujetos que respondieron la encuesta } \\
\text { (n = 9.851). Algunos profesionales marcaron una opción, mientras que otros marcaron dos, por lo que el total } \\
\text { de respuestas es mayor que el total de sujetos. }\end{array}$
\end{tabular}

Tabla V. Marque las dos principales fuentes de información que utiliza más frecuentemente.

\begin{tabular}{lc}
\hline Revista de sociedad científica nacional & $4.499(45,7 \%)$ \\
\hline $\begin{array}{l}\text { Guías clínicas y otras publicaciones distribuidas } \\
\text { por autoridades sanitarias (municipal, provincial, nacional) }\end{array}$ & $3.972(40,3 \%)$ \\
\hline $\begin{array}{l}\text { En Internet: Wikipedia, redes sociales, foros, } \\
\text { publicaciones científicas internacionales, sitios web médicos }\end{array}$ & $1.626(16,5 \%)$ \\
\hline \begin{tabular}{l} 
Documentación que distribuye la industria farmacéutica \\
\hline
\end{tabular}
\end{tabular}

El porcentaje, en cada una de las fuentes de información, corresponde al total de sujetos que respondieron la encuesta $(n=9.851)$. Algunos profesionales marcaron una opción, mientras que otros marcaron dos, por lo que el total de respuestas es mayor que el total de sujetos.

En este estudio, la participación en actividades presenciales, como congresos y cursos en sociedades científicas y universidades, es la modalidad más elegida para mantenerse actualizado. Probablemente, los sujetos que respondieron el cuestionario interpretan que la actualización de conocimientos necesariamente corresponde a una actividad de edu- 
Tabla VI. Combinaciones más frecuentes sobre fuentes de información para actualizar los conocimientos $(n=9.851)$.

\begin{tabular}{lc}
\hline Guías clínicas/Internet & $4.361(44,2 \%)$ \\
\hline Revista de sociedad científica nacional/Internet & $3.047(30,9 \%)$ \\
\hline Revista de sociedad científica nacional/guías clínicas & $1.344(13,6 \%)$ \\
\hline
\end{tabular}

Tabla VII. ¿Cuáles son los recursos de Internet que utiliza con mayor frecuencia?

\begin{tabular}{lc}
\hline Búsqueda bibliográfica en PubMed & $7.410(75,2 \%)$ \\
\hline Sitios web médicos & $7.165(72,7 \%)$ \\
\hline Publicaciones científicas internacionales & $6.383(64,8 \%)$ \\
\hline Wikipedia & $2.001(20,3 \%)$ \\
\hline Foros & $1.597(16,2 \%)$ \\
\hline Redes sociales & $608(6,9 \%)$
\end{tabular}

El total de respuestas es mayor que el total de sujetos. El porcentaje, en cada uno de los recursos, se refiere al total de sujetos que respondieron la encuesta $(n=9.851)$. La mayoría señaló tres o cuatro opciones, y muchos marcaron las seis respuestas posibles, lo que generó gran cantidad de combinaciones.

cación formal y 'teórica'. Según las respuestas obtenidas, la modalidad presencial es la más utilizada, pero en la práctica, en Argentina, ha disminuido notablemente la asistencia a los cursos presenciales organizados por sociedades científicas o por la industria farmacéutica.

La opción de respuesta 'Hacer cursos a distancia con entrega de material impreso en domicilio' se incluyó en el cuestionario porque en Argentina se ofrecen, desde sociedades científicas y desde una editorial de textos médicos, cursos de actualización con modalidad de educación a distancia que distribuyen textos impresos por correo postal. La respuesta obtenida, 'nadie utiliza esa modalidad', resultó una sorpresa porque dichos cursos cuentan con un buen número de profesionales inscritos. En distintos ámbitos se discute la decisión de ofrecer actualizaciones en soporte electrónico o seguir publicando en papel. Por el momento, coexisten ambas modalidades y se advierte un gradual aumento de la oferta de cursos online. Poco menos del $30 \%$ señala que busca información en Internet para actualizar sus conocimientos; un 16\% recurre a Wikipedia, redes sociales y sitios web médicos. En cualquier caso, el uso de Internet en- tre los médicos todavía no es la estrategia de actualización dominante.

Llama la atención que las opciones de 'intercambio entre colegas' y 'consulta a un colega' tengan un bajo porcentaje de respuestas porque en la bibliografía se menciona que la interacción entre colegas es el mecanismo de aprendizaje privilegiado por los médicos. La WFME [4] sostiene que 'generalmente los médicos desarrollan y cambian su práctica mediante intercambio de opiniones entre profesionales, el diálogo con colegas, más que como resultado de procesos educativos formales. Es probable que la interacción entre colegas sea vivida e interpretada como parte de la práctica profesional cotidiana y que no se visualice como una estrategia de actualización.

Las fuentes de información más utilizadas son las revistas publicadas por las sociedades científicas y las guías clínicas y otras publicaciones distribuidas por las autoridades sanitarias (municipales, provinciales o nacionales). Si los profesionales acceden a la información a través de material impreso o de material electrónico no se pudo identificar claramente porque la pregunta estuvo mal formulada y las opciones no eran lo suficientemente específicas. En general, las revistas y los documentos producidos por las autoridades sanitarias están disponibles en formato electrónico. Probablemente algunos profesionales acceden a las revistas y a las guías clínicas vía Internet. Las combinaciones guías clínicas/Internet y revista de sociedad científica nacional/Internet refuerzan esta idea. Las respuestas a la pregunta número 5 también van en esa misma línea: se busca información científica en la web.

En términos generales se podría concluir que las estrategias más utilizadas para actualizar conocimientos son los cursos presenciales y los congresos, la lectura de revistas científicas y la tarea asistencial en el ámbito hospitalario. Pocos mencionaron la interacción entre colegas como un recurso para el aprendizaje.

Se ha demostrado [6-8] que no existe ningún método o herramienta de educación médica continua que por sí solo - particularmente los más usados, como son las conferencias, clases, pósters y simposioscambie la conducta de los médicos, y menos que cambie los resultados de su acción con los pacientes.

El desarrollo profesional continuo [9] es un imperativo profesional de cada médico, un prerrequisito para mejorar la calidad de la atención sanitaria; es una condición necesaria, pero no suficiente, para la mejora de la calidad de los servicios de salud que recibe la población.

Albert Bandura [10], en su obra Pensamiento y acción, enfatiza: 'La adquisición de conocimientos 
y de habilidades referentes a las innovaciones es necesaria, pero no es suficiente para que produzca su adopción en la práctica. Son varios los factores que determinan si el individuo actuará de acuerdo con lo que ha aprendido'.

\section{Bibliografía}

1. Cantillon P, Jones R. Does continuing medical education in general practice make a difference? BMJ 1999; 318: 1276-9.

2. Osler W. An address on the importance of post-graduate study. Br Med J 1900; 2: 73.

3. Mejía A. Educación continua. Educ Med Salud 1986; 20 43-71.

4. World Federation Medical Education (WFME). Desarrollo profesional continuo (DPC) de los médicos. Estándares globales de la WFME para la mejora de calidad. Educ Med 2004; 7 (Supl 2): S1-56.

5. Slotnick HB. Physicians' learning strategies. Chest 2000; 118: S18-23.

6. Mazmanian PE, Davis DA. Continuing medical education and the physician as a learner: guide to the evidence. JAMA 2002; 288: 1057-60.

7. Smith WR. Evidence for the effectiveness of techniques to change physician's behavior. Chest 2000; 118: S8-17.

8. Forsetlund L, Bjørndal A, Rashidian A, Jamtvedt G, O'Brien MA, Wolf $\mathrm{F}$, et al. Continuing education meetings and workshops: effects on professional practice and health care outcomes. Cochrane Database Syst Rev 2009; 2: CD003030.

9. Peck C, McCall M, McLaren B, Rotem T. Continuing medical education and continuing professional development: international comparisons. BMJ 2000; 320: 432-5.

10. Bandura A. Pensamiento y acción. Biblioteca de Psicología, Psiquiatría y Salud. Barcelona: Martínez Roca; 1987. 